\title{
Protective effects of folic acid on colon carcino- genesis induced by azoxymethane in mice
}

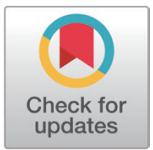

Received: Jun 29, 2021

Revised: Jul 23, 2021

Accepted: Jul 27, 2021

${ }^{*}$ Corresponding author

Hyun Jik Lee

College of Veterinary Medicine and

Veterinary Medicine Center, Chungbuk

National University, Cheongju 28644,

Korea

Tel: +82-43-261-2597

E-mail: leehyunjik@chungbuk.ac.kr

Beom Jun Lee

College of Veterinary Medicine and

Veterinary Medicine Center, Chungbuk

National University, Cheongju 28644,

Korea

Tel: +82-43-261-3357

E-mail: beomjun@cbu.ac.kr

Copyright @ 2021 Research Institute of Veterinary Medicine, Chungbuk National University. This is an Open Access article distributed under the terms of the Creative Commons Attribution Non-Commercial License (http://creativecommons.org/licenses/ by-nc/4.0/) which permits unrestricted non-commercial use, distribution, and reproduction in any medium, provided the original work is properly cited.

ORCID

Ji Yun Sohn

https://orcid.org/0000-0002-3331-5987

Eun Young Kim

https://orcid.org/0000-0002-9972-664X

Jae-Hwang Jeong

https://orcid.org/0000-0003-3920-3799

Dae Joong Kim

https://orcid.org/0000-0003-3564-9478

Sang Yoon Nam

https://orcid.org/0000-0001-7576-6543

\author{
Ji Yun Sohn ${ }^{1}$, Eun Young Kim², Jae-Hwang Jeong ${ }^{3}$, Dae Joong Kim¹, \\ Sang Yoon Nam ${ }^{1}$, Hyun Jik Lee ${ }^{1,4,5^{*}}$, Beom Jun Lee ${ }^{1 *}$ \\ ${ }^{1}$ College of Veterinary Medicine and Veterinary Medicine Center, Chungbuk National University, \\ Cheongju 28644, Korea \\ ${ }^{2}$ Korea Food Culture Promotion Association, Cheongju 28553, Korea \\ ${ }^{3}$ Department of Biotechnology and Biomedicine, Chungbuk Province College, Cheongju 28160, Korea \\ ${ }^{4}$ Laboratory of Veterinary Physiology, College of Veterinary Medicine, Chungbuk National University, \\ Cheongju 28644, Korea \\ ${ }^{5}$ Institute for Stem Cell \& Regenerative Medicine (ISCRM), Chungbuk National University, Cheongju \\ 28644, Korea
}

\begin{abstract}
Colon cancer has been considered a leading cause of cancer-associated death. Folic acid is a vitamin necessary for cellular physiological functions and cell viability. However, the association between folic acid intake and colon cancer has been examined in several prospective cohort studies are controversial. This study investigated the effects of folate intake on colon carcinogenesis and oxidative stress in an azoxymethane (AOM)/dextran sodium sulfate (DSS) institute for cancer research (ICR) mouse model. Thirty male ICR mice (5 weeks old) were divided into the control group and the experimental group supplied $0.03 \%$ folic acid via drinking water (50 mL/week/mouse) for 6 weeks. To induce colonic pre-neoplastic lesions, the animals were subcutaneously injected three times weekly with AOM (10 mg/kg body weight), followed by $2 \%$ DSS in drinking water for a week. Folic acid supplementation significantly suppressed the total number of aberrant crypt foci and aberrant crypts. Histological image data showed that folic acid supplementation attenuated neoplastic change. In addition, we measured the thiobarbituric acid reactive substances concentration of dry feces samples to identify the effect of folic acid on reactive oxygen accumulation. The folic acid supplementation group had reduced reactive oxygen species levels in dry feces compared to the control group. In conclusion, these findings indicate that folic acid suppresses colon carcinogenesis and oxidative stress in an AOM/DSS mouse model.
\end{abstract}

Keywords: folic acid, colonic neoplasms, reactive oxygen species, oxidative stress

\section{INTRODUCTION}

Colon cancer is the third leading cause of cancer-associated deaths worldwide, representing the global health burden in recent years $[1,2]$. Epidemiological and experimental evidence suggested that lifestyle change, such as diet, is a potent factor in colon cancer occurrence [3-5]. Also, many researchers focused on identifying specific nutrients for the development of primary prevention of colon cancer occurrence. Indeed, some researchers have tried to identify the role of nutrients, 
Hyun Jik Lee

https://orcid.org/0000-0002-2762-2649

Beom Jun Lee

https://orcid.org/0000-0002-7013-8086

Conflict of Interest

The authors declare that they have no

conflict of interest.

\section{Acknowledgements}

This research was supported by Chungbuk National University Korea National University Development Project (2020).

Ethics Approval

This article does not require IRB approval because there is no human participants. The animal experiments was conducted in compliance with the IACUC no. CBNUA-1139-18-01. including meat, fat, fiber, and vitamins, in colon carcinogenesis [6-8].

Folate is a water-soluble vitamin B9 enriched in dark leafy vegetables, and folic acid is a synthetic compound used as a vitamin supplement [9]. Also, folate supplementation is required for one-carbon metabolism and DNA biosynthesis in proliferating cells [9]. Intracellular folates act as one-carbon moieties and mediate epigenetic modification, such as DNA, RNA, and histone methylation. Low folate levels in plasma are closely associated with reduced colorectal cancer risk and inhibit the progression of preneoplastic and neoplastic lesions [10]. Several prospective cohort studies have investigated the association between folic acid intake and colon cancer occurrence $[11,12]$. A recent meta-analysis has shown that colon cancer risk is significantly reduced in subjects with the highest quintile of dietary folic acid intake than the lowest intake [12]. However, another meta-analysis study has presented that folic acid supplementation has no effects on colorectal cancer risk [11]. To identify the effects of folic acid supplementation on colon carcinogenesis, further investigation with a rigorous experimental design is needed.

Reactive oxygen species (ROS), such as hydrogen peroxide, hydroxyl radical, and superoxide anion, are produced during normal energy metabolism [13]. They induce oxidative stress, causing DNA strand breaks, lipid peroxidation, and genetic instability, which are closely associated with colorectal carcinogenesis [14]. Many researchers have presented an oxidative stress-based antitumorigenic strategy with antioxidants and hypoxia adaptation [15]. Several studies have reported the protective effects of dietary folic acid on colon cancer development in animal models [16-18]. However, the role of folic acid-regulated ROS in colon carcinogenesis in experimental animal models is unknown. Therefore, this study aimed to investigate the effects of folic acid supplementation on colon neoplastic change and ROS generation in an azoxymethane (AOM)/dextran sodium sulfate (DSS)-induced colon cancer mouse model.

\section{MATERIALS AND METHODS}

\section{Materials}

AOM and folic acid were obtained from Sigma-Aldrich (St. Louis, MO, USA). DSS (molecular weight 36,000-50,000) was obtained from MP Biomedical (Santa Ana, CA, USA).

\section{Animals}

Four-week-old male institute for cancer research (ICR) mice were purchased from Samtako Bio Korea (Osan, Korea) and housed in an isolating mouse cage (five mice per cage). The temperature and humidity were set at $20 \pm 2{ }^{\circ} \mathrm{C}$ and $50 \pm 20 \%$, respectively. Purified diet AIN$76 \mathrm{~A}$ was purchased from Central Laboratory Animal (Seoul, Korea). During the experimental periods, all diets and litter were used after sterilization. The entire animal experiment was performed in compliance with the Guide for Care and Use of Laboratory Animals of Chungbuk National University (Institutional Animal Care and Use Committee [IACUC] approval no. CBNUA-1139-18-01). Body weight and food consumption were recorded weekly. 


\section{Experimental design}

After acclimation to the cage for 1 week, 30 mice ( 5 weeks old) were divided into control and experimental groups. AIN-76A diet and distilled water were supplied to mice. Folic acid $(0.03 \%)$ was supplemented via drinking water $(50 \mathrm{~mL} / \mathrm{week} / \mathrm{mouse})$ to the experimental group. To induce the formation of preneoplastic lesions in the colon, AOM $(10 \mathrm{mg} / \mathrm{kg}$ body weight) was subcutaneously injected thrice at 0,1 , and 2 weeks of the experimental period. At the third week of the experimental period, distilled water with 2\% DSS was provided for 7 days. The total experimental period was 6 weeks. At the termination of the study, mice were sacrificed by cervical dislocation to examine the colon. After mice were sacrificed, entire large intestine samples were collected. Feces were obtained in the cage before sacrifice. Large intestine samples were washed with saline and fixed in $10 \%$ neutral buffered formalin. The detailed experimental design is described in Fig. 1.

\section{Assay of aberrant crypt (AC) foci (ACF)}

Colons were collected and rinsed with $0.9 \% \mathrm{NaCl}$ solution. Colons were then fixed flat in $10 \%$ neutral phosphate-buffered formalin. Fixed colons were stained with $0.2 \%$ methylene blue for $2 \mathrm{~s}$. Colon samples were placed on the mucosal surfaces of a slide for examination under a light microscope. The total numbers of $\mathrm{ACF}$ and $\mathrm{AC}$ in each focus were counted under a microscope $(\times 40$ and $\times 100$; Fig. 2$)$.

\section{Histopathological examination}

Tissue samples were fixed in 10\% neutral buffered formalin. Fixed tissue was paraffin-embedded and then cut to multiple $4 \mu \mathrm{m}$ sections. Sliced samples were stained with hematoxylin and eosin (H \& E) for histopathological examination under a light microscope (Olympus, Tokyo, Japan).

\section{Thiobarbituric acid reactive substance (TBARS) assay}

The dry feces of five mice were diluted with distilled water and then incubated at $37^{\circ} \mathrm{C}$ for 1 h. The samples were centrifuged for $15 \mathrm{~min}$ at $15,000 \times \mathrm{g}$, and the supernatant was collected.



\begin{tabular}{|c|c|c|c|}
\hline Group & No. of: & & \\
\hline Control & 15 & AIN-76A diet & \\
\hline Folic acid & 15 & AIN-76A diet & $0.03 \%$ Folic acid \\
\hline
\end{tabular}

Fig. 1. Experimental design for azoxymethane (AOM)/dextran sodium sulfate (DSS)-induced colon carcinogenesis in mice. AOM (10 mg/kg body weight in saline, subcutaneously, thrice weekly), DSS ( $2 \%$ in distilled water for 1 week), and folic acid ( $0.03 \%$ in distilled water ad libitum). 


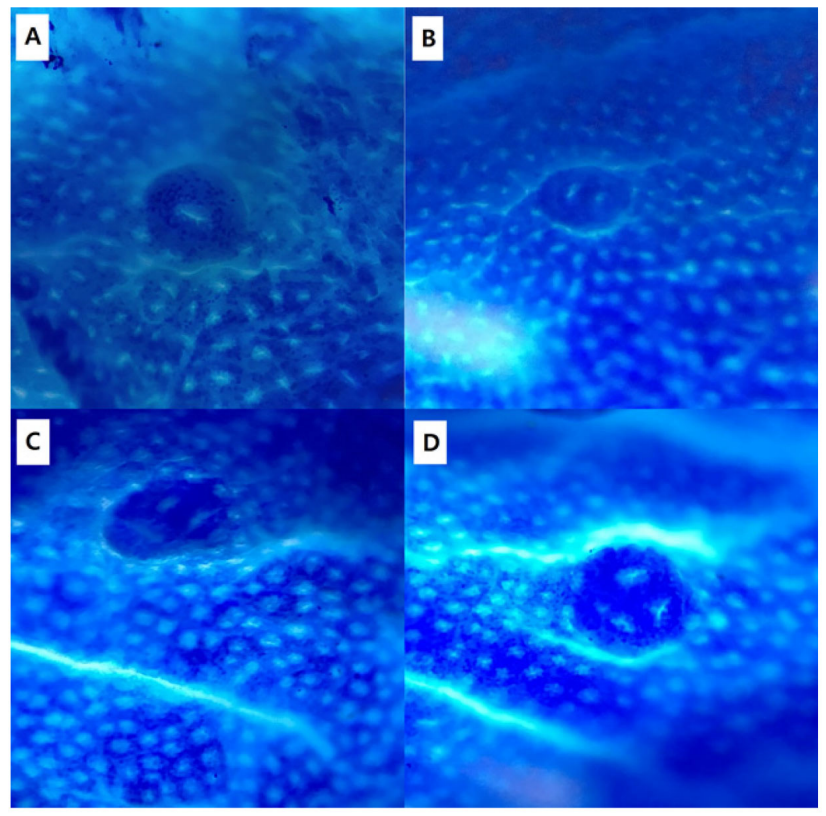

Fig. 2. Colonic aberrant crypt foci (ACF) images. (A-D) ACF were stained with methylene blue and visualized under a light microscope $(\times 40)$.

Malondialdehyde (MDA) levels were detected using the TBARS Assay Kit (Cayman Chemical, Ann Arbor, MI, USA). The assay was carried out according to the supplier's instructions.

\section{Statistical analysis}

All statistical analyses were performed using GraphPad Prism 5 software (GraphPad, San Diego, CA, USA). Comparisons of two groups were performed using Student's $t$-test. Data were expressed as the mean \pm S.D. Significant differences were determined at the level of $p<0.05$ or $p<0.01$.

\section{RESULTS}

\section{Effects of folic acid on body weight and histological changes in an azoxymethane (AOM)/dextran sodium sulfate (DSS) mouse model}

To identify the effects of folic acid supplementation on colon carcinogenesis of the AOM/ DSS mouse model, the body weight of an AOM/DSS mouse model was investigated weekly. All mice grew at a similar rate throughout the study. During the entire experiment, the control group were heavier than the folic acid supplementation group (Fig. 3). At the end of the experiment, the final weight of the two groups was not significantly different (Fig. 3). Also, H \& E staining for colon epithelium was performed to identify the effects of folic acid supplementation on histological changes in an AOM/DSS mouse model. As shown in Fig. 4, the folic acid supplementation group revealed ameliorated hyperplastic change in colonic mucosa compared to the control group. Concerning that $\mathrm{ACF}$ and $\mathrm{AC}$ formations are considered the earliest morphological changes during colon carcinogenesis, the modulatory effects of folic acid on ACF and $\mathrm{AC}$ formation in colon tissue were checked. The total numbers of $\mathrm{ACF}$ and $\mathrm{AC}$ lesions 


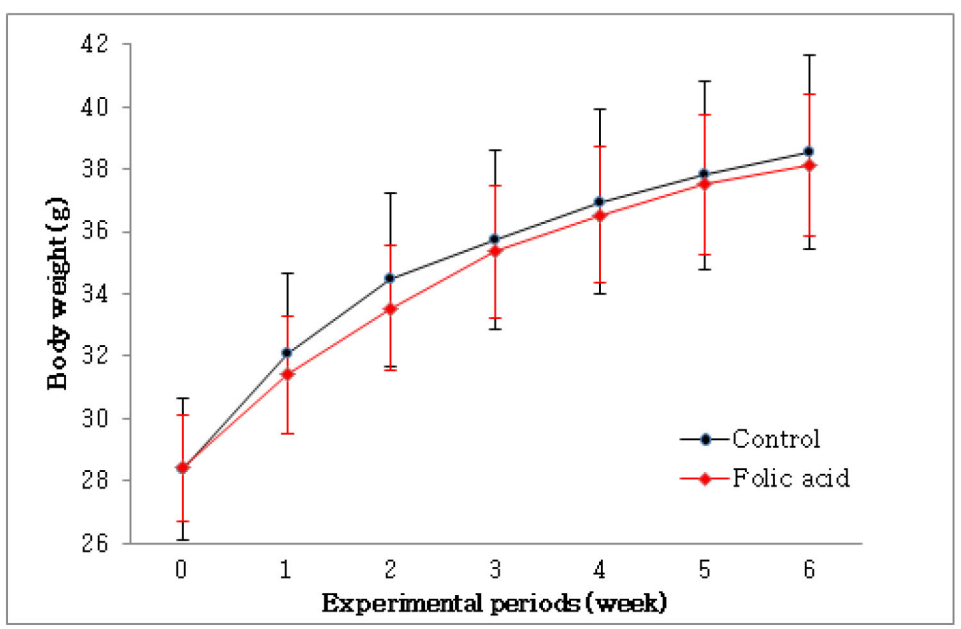

Fig. 3. Changes in body weight of mice treated with azoxymethane (AOM)/dextran sodium sulfate (DSS) and folic acid. The body weight of the two groups increased as time goes on. Data are mean \pm S.D. ( $n=$ 15).

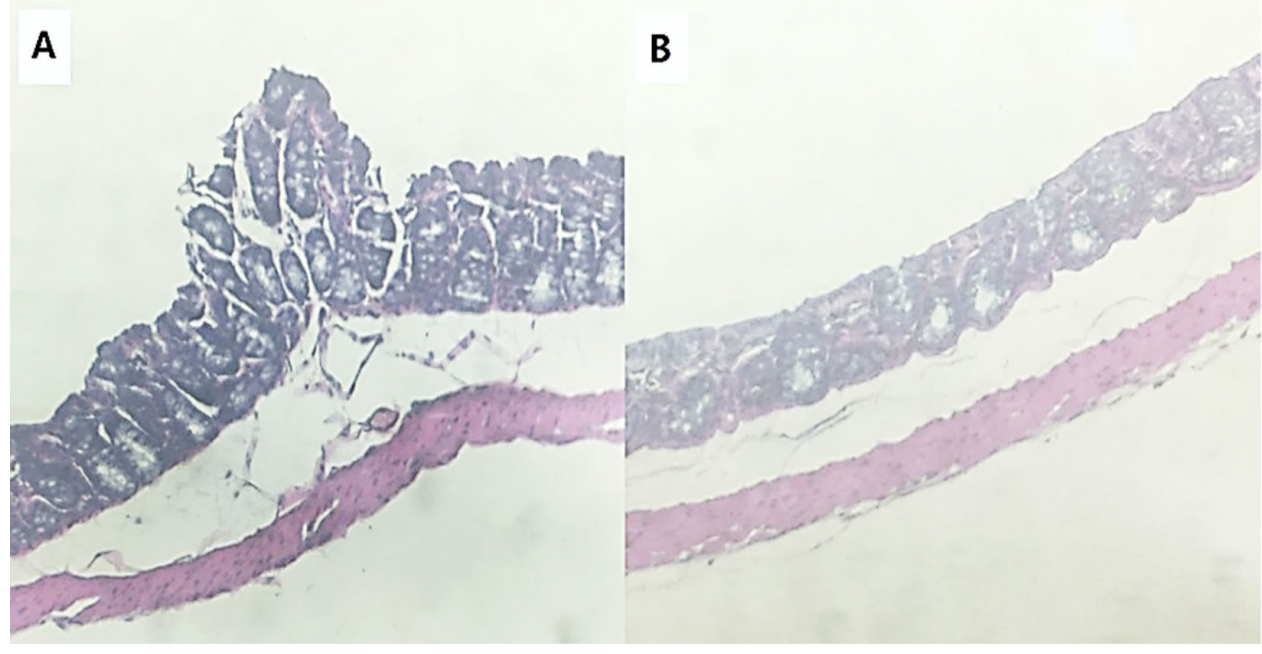

Fig. 4. Histopathology of colonic epithelium in the control group. Histological images of colonic epithelium in the control group (A) and the folic acid supplementation group (B) were visualized by hematoxylin and eosin staining under a light microscope $(\times 100)$.

in colon tissue of an AOM/DSS mouse model were measured. As shown in Figs. 5 and 6, the total number of $\mathrm{ACF}$ in the folic acid supplementation group (5.7 ACFs) decreased compared to controls (9.3 ACFs). Also, the total number of $\mathrm{AC}$ in the folic acid supplementation group (7.5 ACs) decreased compared to the control group (12.4 ACs). These data indicated folic acid supplementation suppresses hyperplastic alteration in colon tissue of an AOM/DSS mouse model.

\section{Antioxidative effects of folic acid on an azoxymethane (AOM)/dextran sodium sul-} fate (DSS) mouse model

MDA concentrations in dry feces have been considered markers for oxidative stressstimulated lipid peroxidation in the colon mucosa. To investigate the regulatory effects of folic acid supplementation on oxidative stress of an AOM/DSS mouse model, a TBARS 


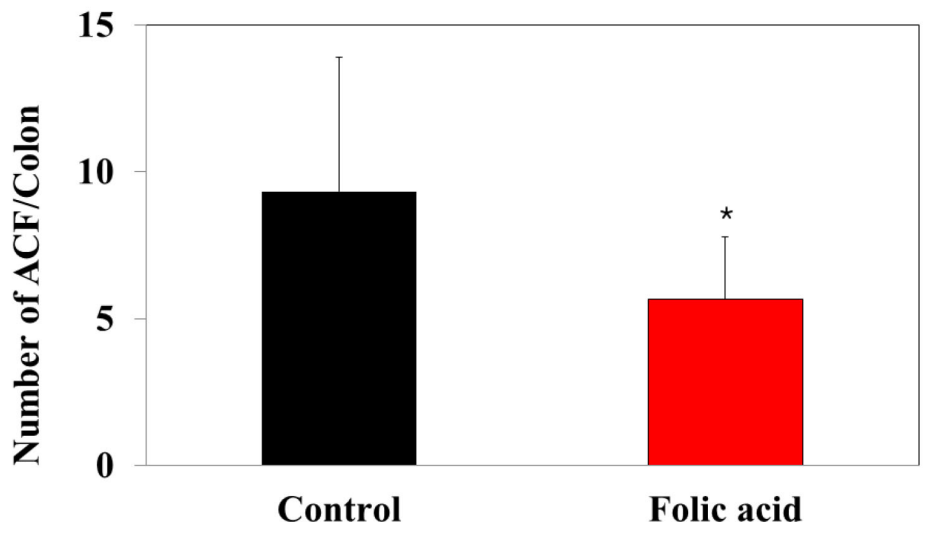

Fig. 5. Effects of folic acid on the formation of colonic ACF in mice. Data are the mean \pm S.D. $(n=15)$. * Significantly different from the control group $(p<0.05)$. ACF, aberrant crypt foci.

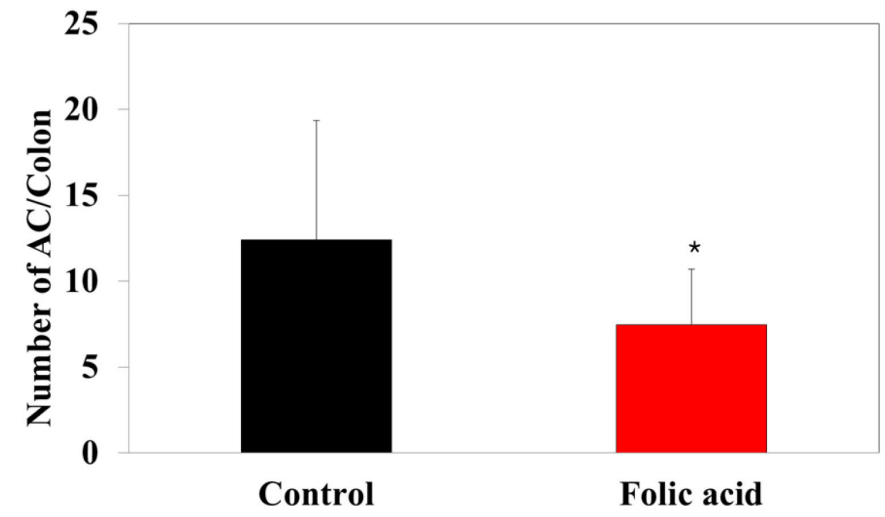

Fig. 6. Effects of folic acid on the formation of colonic aberrant crypt (AC) in AOM/DSS mice. Data are the mean \pm S.D. $(n=15)$. ${ }^{*}$ Significantly different from the control group $(p<0.01)$. AOM, azoxymethane; DSS, dextran sodium sulfate.

assay was performed with dry feces samples. The TBARS concentration in the folic acid supplementation group $(8.3 \mu \mathrm{M})$ significantly decreased compared to controls $(16.6 \mu \mathrm{M}$; Fig. 7). This observation revealed the antioxidative effects of folic acid supplementation on an AOM/DSS mouse model, suggesting the possibility of close relationships between folic acid antioxidation and colon carcinogenesis.

\section{DISCUSSION}

This study demonstrated that folic acid supplementation suppresses AOM/DSS-induced colon carcinogenesis and ROS accumulation in an in vivo model. AOM is a very potent carcinogen that induces colon cancer with a high incidence in experimental animals [19, 20]. AOM-induced methyl diazonium ion is a highly reactive electrophile that increases oxidative stress in the liver and colon tissue [21]. Also, AOM induces ACFs that are incipient lesions in colon cancer development [22]. ACF is identified by microscopy on methylene bluestained colon mucosa and characterized by enlarged and risen dark crypts [23]. Therefore, 




Fig. 7. Effects of folic acid on lipid peroxidation in the feces of an AOM/DSS mouse model. The lipid peroxidation levels of dry feces samples were measured by TBARS assay. Data are the mean \pm S.D. $(n=$ 15). * Significantly different from the control group $(p<0.01)$. AOM, azoxymethane; DSS, dextran sodium sulfate; TBARS, thiobarbituric acid reactive substance.

ACFs are used as colon cancer biomarkers [24]. This study also found histological alterations, including AC and ACF formations with hyperplastic change, in an AOM/DSS-induced colon carcinogenic mouse model.

Although some meta-analysis reports suggested that folate supplementation does not affect colorectal cancer risk, many researchers reported the therapeutic effects of folic acid on various types of cancers, including colorectal cancer [10, 11, 25, 26]. Also, targeting the folic acid receptor $\alpha$ has been considered a promising targeted therapeutic strategy in oncology [27]. Consistent with these findings, the protective effects of folic acid on an AOM/DSS mouse model are presented.

Present study showed that folic acid supplementation significantly alleviated the hemininduced ROS generation in colon carcinogenesis mouse model. Elevated ROS levels have been well documented in various types of cancers [28-30]. Furthermore, oxidative stressinduced DNA damage causes colorectal carcinogenesis by genetic mutation [13]. Previous reports suggested that the protective effects of folic acid are related to the methionine cycle. In the methionine cycle, homocysteine derived from methionine contributes to cysteine formation, which is essential for the formation of the antioxidant molecule glutathione (GSH) [31]. Folate-regulated GSH expression has a critical role in redox homeostasis [31]. Consistent with these findings, many reports showed evidence supporting the antioxidative effects of folic acid supplementation. In an AOM-induced colon carcinogenesis rat model, folate and vitamin $B_{12}$ supplementation alleviates AOM-stimulated GSH depletion and protein oxidation and DNA oxidative damage [32]. One report showed that a folate-deficient tumor microenvironment increased ROS and metastasis but decreased proliferation in human hepatocellular carcinoma [26]. Also, tert-butyl hydroperoxide-induced oxidative stress decreased folic acid uptake by suppressing folate transporter mRNA, which plays a key role in the proliferation and viability of colorectal cancer cells [33]. These and previous findings indicated that folic acid-suppressed oxidative stress plays a key role in preventing ROS-induced colon carcinogenesis through the regulation of genetic mutation and cancer 
pathogenesis.

In conclusion, the results have shown that folic acid prevents ROS accumulation and colon carcinogenesis, although most antitumorigenic therapeutic strategies employ oxidative stress to disturb the cellular redox state and cancer cell death [13]. Considering that colon carcinogenesis in an AOM/DSS mouse model is related to excessive oxidative stress, this study suggests folic acid supplementation therapy as a preventive strategy for patients with a high risk of colon cancer.

\section{REFERENCES}

1. Seiwert N, Heylmann D, Hasselwander S, Fahrer J. Mechanism of colorectal carcinogenesis triggered by heme iron from red meat. Biochim Biophys Acta Rev Cancer 2020;1873:188334.

2. Peto J. Cancer epidemiology in the last century and the next decade. Nature 2001;411:390-395.

3. Gamage SMK, Dissabandara L, Lam AKY, Gopalan V. The role of heme iron molecules derived from red and processed meat in the pathogenesis of colorectal carcinoma. Crit Rev Oncol Hematol 2018;126:121-128.

4. Wiseman M. The second World Cancer Research Fund/American Institute for Cancer Research expert report. Food, nutrition, physical activity, and the prevention of cancer: a global perspective. Proc Nutr Soc 2008;67:253-256.

5. Ronellenfitsch U, Kyobutungi C, Ott JJ, Paltiel A, Razum O, Schwarzbach M, Winkler V, Becher H. Stomach cancer mortality in two large cohorts of migrants from the Former Soviet Union to Israel and Germany: are there implications for prevention? Eur J Gastroenterol Hepatol 2009;21:409-416.

6. Willett WC, Stampfer MJ, Colditz GA, Rosner BA, Speizer FE. Relation of meat, fat, and fiber intake to the risk of colon cancer in a prospective study among women. N Engl J Med 1990;323:1664-1672.

7. Meyerhardt JA, Niedzwiecki D, Hollis D, Saltz LB, Hu FB, Mayer RJ, Nelson H, Whittom R, Hantel A, Thomas J, Fuchs CS. Association of dietary patterns with cancer recurrence and survival in patients with stage III colon cancer. JAMA 2007;298:754-764.

8. Dulai PS, Singh S, Marquez E, Khera R, Prokop LJ, Limburg PJ, Gupta S, Murad MH. Chemoprevention of colorectal cancer in individuals with previous colorectal neoplasia: systematic review and network meta-analysis. BMJ 2016;355:16188.

9. Locasale JW. Serine, glycine and one-carbon units: cancer metabolism in full circle. Nat Rev Cancer 2013;13:572-583.

10. Gylling B, Van Guelpen B, Schneede J, Hultdin J, Ueland PM, Hallmans G, Johansson I, Palmqvist R. Low folate levels are associated with reduced risk of colorectal cancer in a population with low folate status. Cancer Epidemiol Biomark Prev 2014;23:2136-2144.

11. Qin T, Du M, Du H, Shu Y, Wang M, Zhu L. Folic acid supplements and colorectal cancer risk: meta-analysis of randomized controlled trials. Sci Rep 2015;5:12044.

12. Sanjoaquin MA, Allen N, Couto E, Roddam AW, Key TJ. Folate intake and colorectal cancer risk: a meta-analytical approach. Int J Cancer 2005;113:825-828. 
13. Sreevalsan S, Safe S. Reactive oxygen species and colorectal cancer. Curr Colorectal Cancer Rep 2013;9:350-357.

14. Saud SM, Li W, Morris NL, Matter MS, Colburn NH, Kim YS, Young MR. Resveratrol prevents tumorigenesis in mouse model of Kras activated sporadic colorectal cancer by suppressing oncogenic Kras expression. Carcinogenesis 2014;35:2778-2786.

15. Carini F, Mazzola M, Rappa F, Jurjus A, Geagea AG, Al Kattar S, Bou-Assi T, Jurjus R, Damiani P, Leone A, Tomasello G. Colorectal carcinogenesis: role of oxidative stress and antioxidants. Anticancer Res 2017;37:4759-4766.

16. Kim YI, Salomon RN, Graeme-Cook F, Choi SW, Smith DE, Dallal GE, Mason JB. Dietary folate protects against the development of macroscopic colonic neoplasia in a dose responsive manner in rats. Gut 1996;39:732-740.

17. Song J, Sohn KJ, Medline A, Ash C, Gallinger S, Kim YI. Chemopreventive effects of dietary folate on intestinal polyps in Apc+/-Msh2-/- mice. Cancer Res 2000;60:3191-3199.

18. Song J, Medline A, Mason JB, Gallinger S, Kim YI. Effects of dietary folate on intestinal tumorigenesis in the apcMin mouse. Cancer Res 2000;60:5434-5440.

19. Ohishi T, Kishimoto Y, Miura N, Shiota G, Kohri T, Hara Y, Hasegawa J, Isemura M. Synergistic effects of (-)-epigallocatechin gallate with sulindac against colon carcinogenesis of rats treated with azoxymethane. Cancer Lett 2002;177:49-56.

20. Sengupta A, Ghosh S, Das S. Modulatory influence of garlic and tomato on cyclooxygenase-2 activity, cell proliferation and apoptosis during azoxymethane induced colon carcinogenesis in rat. Cancer Lett 2004;208:127-136.

21. Sohn OS, Ishizaki H, Yang CS, Fiala ES. Metabolism of azoxymethane, methylazoxymethanol and N-nitrosodimethylamine by cytochrome P450IIE1. Carcinogenesis 1991;12:127-131.

22. Pretlow TP, Barrow BJ, Ashton WS, O'Riordan MA, Pretlow TG, Jurcisek JA, Stellato TA. Aberrant crypt foci: putative preneoplastic foci in human colonic mucosa. Cancer Res 1991;51:1564-1567.

23. Bird RP. Observation and quantification of aberrant crypts in the murine colon treated with a colon carcinogen: preliminary findings. Cancer Lett 1987;37:147-151.

24. Bird RP, Good CK. The significance of aberrant crypt foci in understanding the pathogenesis of colon cancer. Toxicol Lett 2000;112-113:395-402.

25. Hansen MF, Greibe E, Skovbjerg S, Rohde S, Kristensen ACM, Jensen TR, Stentoft C, Kjaer $\mathrm{KH}$, Kronborg CS, Martensen PM. Folic acid mediates activation of the pro-oncogene STAT3 via the folate receptor alpha. Cell Signal 2015;27:1356-1368.

26. Su YH, Huang WC, Huang TH, Huang YJ, Sue YK, Huynh TT, Hsiao M, Liu TZ, Wu ATH, Lin CM. Folate deficient tumor microenvironment promotes epithelial-to-mesenchymal transition and cancer stem-like phenotypes. Oncotarget 2016;7:33246-33256.

27. Cheung A, Bax HJ, Josephs DH, Ilieva KM, Pellizzari G, Opzoomer J, Bloomfield J, Fittall M, Grigoriadis A, Figini M, Canevari S, Spicer JF, Tutt AN, Karagiannis SN. Targeting folate receptor alpha for cancer treatment. Oncotarget 2016;7:52553-52574.

28. Afanas'ev I. Reactive oxygen species signaling in cancer: comparison with aging. Aging Dis 2011;2:219-230. 
29. Kumar B, Koul S, Khandrika L, Meacham RB, Koul HK. Oxidative stress is inherent in prostate cancer cells and is required for aggressive phenotype. Cancer Res 2008;68:1777-1785.

30. Vaquero EC, Edderkaoui M, Pandol SJ, Gukovsky I, Gukovskaya AS. Reactive oxygen species produced by NAD(P)H oxidase inhibit apoptosis in pancreatic cancer cells. J Biol Chem 2004;279:34643-34654.

31. Frye RE, James SJ. Metabolic pathology of autism in relation to redox metabolism. Biomark Med 2014;8:321-330.

32. Padmanabhan S, Waly MI, Taranikanti V, Guizani N, Ali A, Rahman MS, Al-Attabi Z, AlMalky RN, Al-Maskari SNM, Al-Ruqaishi BRS, Dong J, Deth RC. Folate/vitamin B 12 $_{12}$ supplementation combats oxidative stress-associated carcinogenesis in a rat model of colon cancer. Nutr Cancer 2019;71:100-110.

33. Couto MR, Gonçalves P, Catarino T, Araújo JR, Correia-Branco A, Martel F. The effect of oxidative stress upon the intestinal uptake of folic acid: in vitro studies with Caco-2 cells. Cell Biol Toxicol 2012;28:369-381. 